\title{
Acute Lymphoblastic Leukemia Relapse Limited to the Anterior Chamber of the Eye and Treated with Novel CAR T-Cell Therapy
}

\author{
Nir Gomel ${ }^{a}$ Eliya Levinger ${ }^{a}$ Ron Ram ${ }^{b}$ Dror Limon ${ }^{c}$ \\ Zohar Habot-Wilner ${ }^{a}$ \\ aDivision of Ophthalmology, Tel Aviv Sourasky Medical Center, Sackler Faculty of Medicine, \\ Tel Aviv University, Tel Aviv, Israel; 'b Bone Marrow Transplant Unit, Tel Aviv Sourasky \\ Medical Center, Sackler Faculty of Medicine, Tel Aviv University, Tel Aviv, Israel; 'Institute \\ of Radiotherapy, Tel Aviv Sourasky Medical Center, Sackler Faculty of Medicine, Tel Aviv \\ University, Tel Aviv, Israel
}

\section{Keywords}

Acute lymphoblastic leukemia - Anterior chamber - Hypopyon - Chimeric antigen receptor $\mathrm{T}$ cell $\cdot$ Eye $\cdot$ Ocular oncology

\begin{abstract}
Acute lymphoblastic leukemia (ALL) is a malignant neoplasm of the blood stem cells, characterized by increased formation of immature lymphocytes. Ocular manifestations may vary with ocular, adnexal, and orbital involvement. In this case report, we describe the first case of extramedullary relapse of ALL limited to the anterior chamber of the eye treated with the novel chimeric antigen receptor $\mathrm{T}$ (CAR T)-cell therapy and provide a literature review of cases of ALL relapse in the anterior chamber. A 21-year-old male with a history of B-cell ALL presented with a unilateral blurry vision in his left eye. Ocular examination revealed the presence of cells +3 in the anterior chamber and a $1.5-\mathrm{mm}$ hypopyon. Anterior chamber aspiration confirmed a B-ALL relapse. The patient was successfully treated with radiotherapy of his left eye and received CTL-019 transduced T cells (tisagenlecleucel; Novartis) with cytarabine as a bridging chemotherapy treatment. On the last examination, 18 months after the first presentation, the patient presented a complete ocular remission with no systemic or CNS involvement. ALL relapse may involve the anterior chamber of the eye, and an accurate diagnosis is crucial to enable a fast and appropriate treatment. Novel CAR T-cell immunotherapy, combined with ocular irradiation, may be considered in such cases.
\end{abstract}

\section{Karger"}




\section{Introduction}

Acute lymphoblastic leukemia (ALL) is a malignant neoplasm of the blood stem cells, characterized by increased formation of immature lymphocytes. ALL is classified into 2 main subtypes: B-cell and T-cell ALL, in which immature B/T-cell lymphocytes are formed. Treatment usually consists of intravenous chemotherapy with or without intrathecal chemotherapy and irradiation courses. Relapse may occur in up to $20 \%$ of patients $[1,2]$.

Ocular manifestations are formed either from direct leukemic infiltration or indirect due to anemia, thrombocytopenia, and hyperviscosity [3]. The disease may involve the eye, orbit, or adnexa. The anterior chamber can present with uveitis, hyphema, and pseudo-hypopyon. The retina may present with variant hemorrhages, infiltrates, vascular occlusions, and detachment $[3,4]$. Generally, the eye is considered a pharmacological sanctuary for systemic chemotherapy, and thus irradiation is used for infiltration of the eyes and the orbit in addition to chemotherapy $[5,6]$.

Chimeric antigen receptor T (CAR T) cell is a novel immunotherapy treatment, in which engineered $\mathrm{T}$ cells eliminate specific tumor cells. Although gaining popularity, this new technique is not commonly used. First, blood is withdrawn from the patient, lymphopheresis is performed, and T cells are then extracted. These T cells are genetically modified and become chimeric T-cell receptors, comprising antigen-binding site and T-cell activation mechanism. Finally, the CAR T cells are injected back to the patient and target specific antigens on the patient's tumor cells. Tisagenlecleucel (Kymriah; Novartis), anti-CD19-CAR $\mathrm{T}$ cell, has been commercially approved for the treatment of relapse/refractory ALL up to the age of 25 years [7].

We describe the first case of ALL relapse limited to the anterior chamber of the eye which was treated successfully with the novel CAR T-cell immunotherapy in combination with ocular irradiation. In addition, we present a literature review of ALL cases that showed relapse in the anterior chamber of the eye.

\section{Case Report}

A 21-year-old male with a history of B-cell ALL with central nervous system (CNS) involvement was referred to the ophthalmology clinic due to blurry vision in his left eye, 4 months after a bone marrow transplantation (BMT). The patient was diagnosed with Philadelphia-negative B-ALL 1 year prior to his referral and was treated for 4 months with Berlin, Frankfurt, and Muenster protocol consisting of vincristine, daunorubicin, cyclophosphamide, cytarabine, prednisone, asparaginase, and 6-mercaptopurine. Two months after treatment cessation, he underwent a bone marrow aspiration that showed a measurable residual disease. He was treated with blinatumumab - a CD3/CD19 bispecific antibody - for 28 days to eliminate all residual disease and underwent a successful BMT from his sibling. The patient achieved a complete remission to allogeneic hematopoietic cell transplantation.

Twelve months after initial diagnosis and 4 months after BMT, the patient displayed unilateral blurry vision in his left eye. His best-corrected visual acuity (BCVA) was 20/20, and intraocular pressure was $12 \mathrm{~mm} \mathrm{Hg}$ in both eyes. Clinical examination of the right eye (RE) was normal. Left eye (LE) examination revealed cells +3 in the anterior chamber and a 1.5-mm hypopyon (Fig. 1). The lens and the vitreous were clear. A funduscopic examination revealed normal appearance of the macula and retina.

A diagnostic aspiration from the left anterior chamber was performed. Preliminary smear revealed blasts, and a flow cytometry test revealed abnormal CD19 population comprising $98 \%$ of the eye fluid compatible with B-ALL (Fig. 2). A diagnosis of an ocular relapse of B-ALL 


\section{Case Reports in Ophthalmology}

Gomel et al.: Chimeric Antigen Receptor T-Cell Therapy for ALL Relapse in the Anterior Chamber

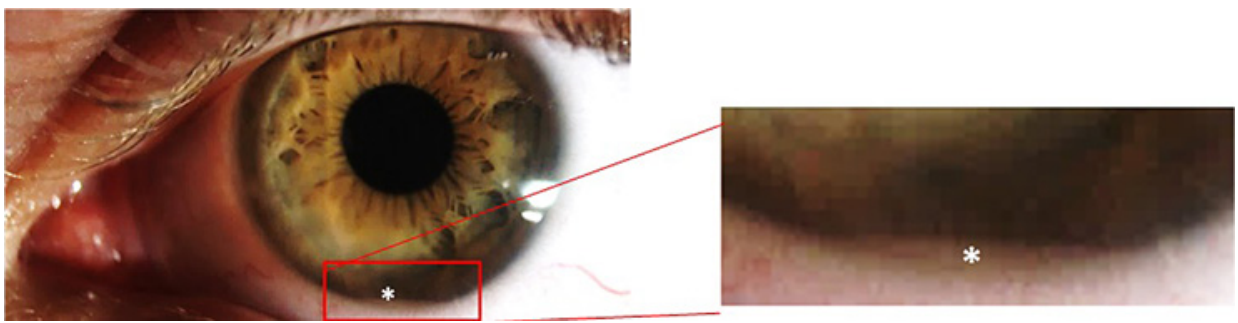

Fig. 1. A color picture of the anterior segment of the left eye demonstrating hypopyon. White asterisk = hypopyon level.

Fig. 2. Flow cytometry test results. The abnormal CD19 population comprising $98 \%$ of the eye fluid.

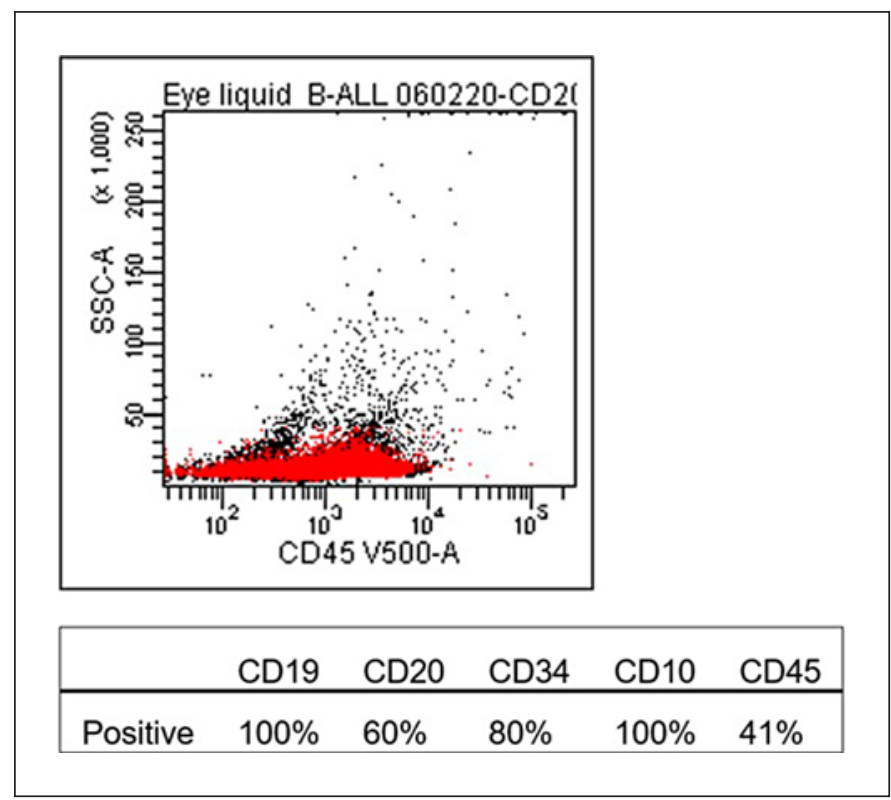

was established. Two days later, the patient underwent a lumbar puncture that was found normal with no evidence for blast cells. Magnetic resonance imaging of the brain was normal with no brain tissue involvement, assuring no systemic or CNS involvement. Bone marrow aspiration excluded recurrence of the disease, and a whole marrow chimerism showed $100 \%$ of donor cells.

In consultation with the hemato-oncologist and radiotherapist and due to of the fact this was an isolated ocular relapse of B-ALL, the patient was referred for an eye irradiation (23.4 Gy divided into 13 daily fractions), bridging with cytarabine chemotherapy to prevent dissemination of leukemia and CAR T-cell therapy. Six days after diagnosis, the patient started receiving radiotherapy for the left eye. Four days later, the patient underwent apheresis treatment for lymphocyte extraction for CAR T-cell preparation.

On examination, 9 days after radiotherapy initiation, the BCVA was 20/20 in both eyes. RE examination was normal. LE examination revealed a clear anterior chamber with no cells or hypopyon (Fig. 3), the lens and vitreous were clear, and fundoscopic examination demonstrated a normal macula and retina.

The patient completed cytarabine bridging chemotherapy treatment, and 2 months after diagnosis (1 month after irradiation), he was admitted for CAR T-cell therapy and received 
Gomel et al.: Chimeric Antigen Receptor T-Cell Therapy for ALL Relapse in the Anterior Chamber

Fig. 3. A color picture of the anterior segment of the left eye 9 days after irradiation initiation demonstrating a clear anterior chamber.

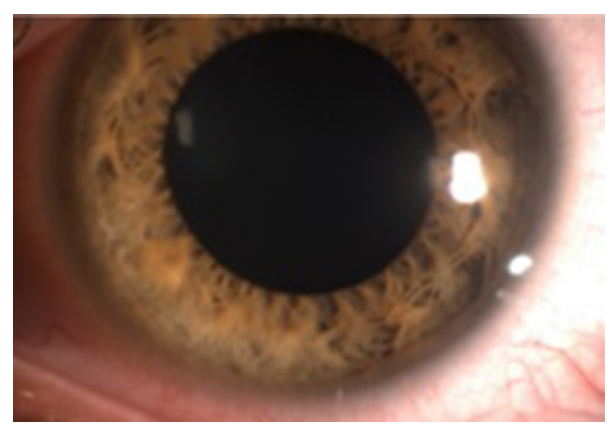

Fig. 4. A color picture of the left eye demonstrating a mild swollen eyelid and a mild enophthalmos.

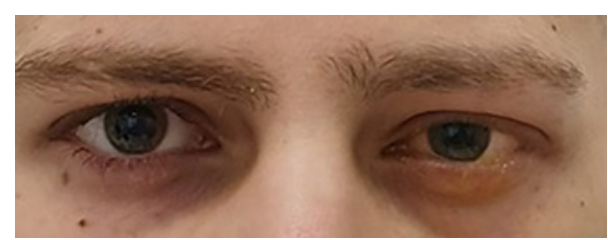

CTL-019 transduced T cells (tisagenlecleucel, Kymriah; Novartis). Two days after treatment, the patient developed neutrophil aplasia and was treated with Neupogen with no hemorrhagic complications. He did not develop cytokine release syndrome or neurologic toxicity.

Ocular examination, 8 days after CAR T infusion and 2 months after irradiation, revealed a mild swollen eyelid and a mild enophthalmos of the LE. These were considered to be related to the radiotherapy (Fig. 4). No ocular movement restriction or diplopia was reported.

Three months after CAR T-cell therapy, the patient presented with few small macular hemorrhages in both eyes and disc hemorrhage in his right eye (Fig. 5). Complete blood count revealed low levels of hemoglobin $(8.5 \mathrm{~g} / \mathrm{dL})$ and platelets $(59,000 / \mu \mathrm{L})$.

A month later and 4 months after CAR T-cell therapy, the patient's BCVA was 20/20 in both eyes. The only finding was a mild swollen upper eye lid of the left eye. All retinal hemorrhages completely absorbed in both eyes, and there was no evidence of ocular B-ALL recurrence in the LE (no cells were found in clinical examination).

At the last follow-up, 18 months after the initial ocular relapse and 12 months after completing CAR T-cell therapy, BCVA was 20/20 in both eyes, and the only clinical finding was a mild swollen upper eyelid of the LE (Fig. 6). There was no systemic or ocular disease relapse.

\section{Discussion}

This is the first case to describe a combined treatment of the novel CAR T-cell treatment with ocular irradiation for ALL relapse in the anterior chamber of the eye. The patient, a 21-year-old male, presented with anterior chamber cells and pseudo-hypopyon in his left eye, 4 months after completing chemotherapy and a BMT. Aspiration from the anterior chamber confirmed an ocular B-ALL relapse. Systemic recurrence was ruled out.

Recurrence may occur with currently available therapies in up to $20 \%$ of ALL patients [1]. To date, a limited number of case series described ALL relapse in the anterior chamber (Table 1) $[8,9,12,14]$. In these previous publications, most patients presented with pseudohypopyon or cells in the anterior chamber within several months up to few years after completing chemotherapy, radiotherapy, or BMT. Most ocular relapses reported were 


\section{Case Reports in Ophthalmology}

\begin{tabular}{l|l}
\hline Case Rep Ophthalmol 2021;12:994-1001 \\
\hline DOI: 10.1159/000521200 & $\begin{array}{l}\text { @ 2021 The Author(s). Published by S. Karger AG, Basel } \\
\text { www.karger.com/cop }\end{array}$ \\
\hline
\end{tabular}

Gomel et al.: Chimeric Antigen Receptor T-Cell Therapy for ALL Relapse in the Anterior Chamber
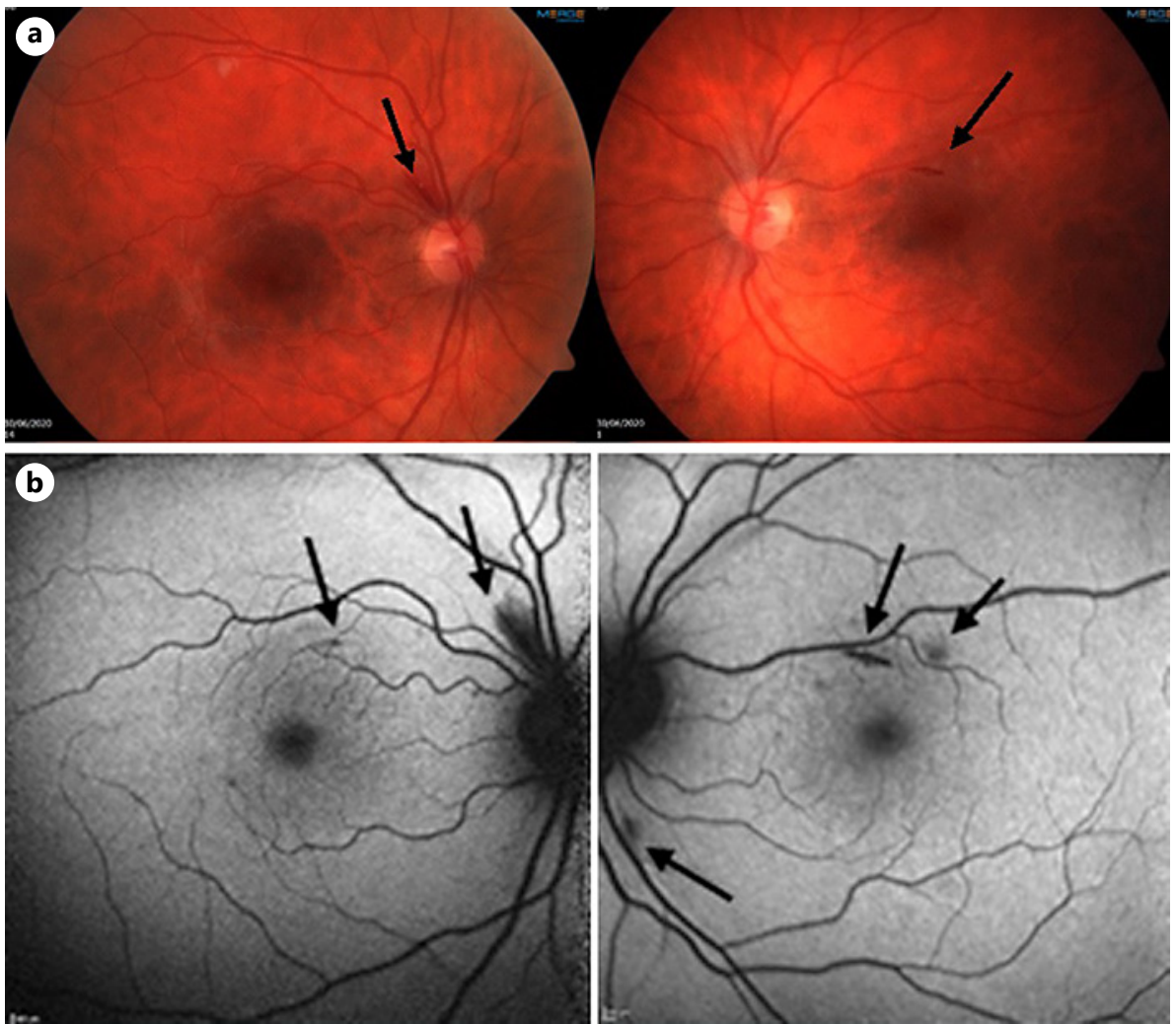

Fig. 5. A color picture (a) and fundus autofluorescence (b) of both eyes demonstrating retinal hemorrhages in both eyes (and disc hemorrhage in the right eye). Black arrows indicate the bleedings.

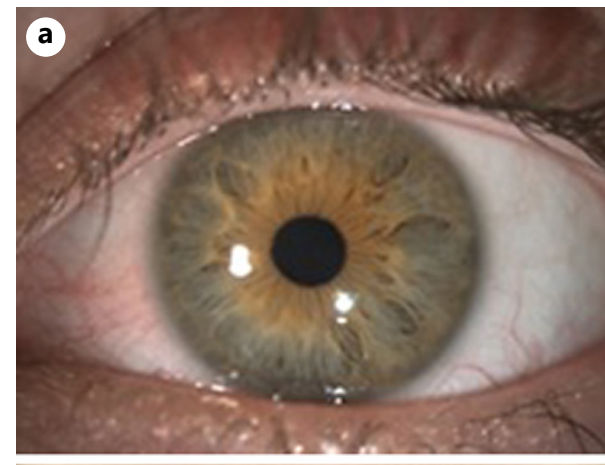

Fig. 6. A color picture of the left eye at the last follow-up (anterior chamber (a) and eyelids (b)). The only finding is a mild swollen upper eyelid.

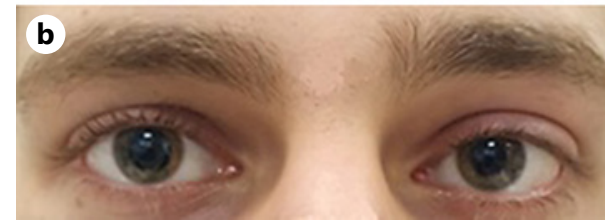

unilateral, whereas only few patients had a bilateral disease. The most common treatment was the combination of chemotherapy and radiotherapy or chemotherapy alone. Two patients were also treated with BMT. Follow-up time was within few months to several years with most reports specifying at least 1-2 years of follow-up. Mortality in cases of ocular relapse limited to the anterior chamber was reported in $40 \%$ of patients. Visual outcomes were not 
Gomel et al.: Chimeric Antigen Receptor T-Cell Therapy for ALL Relapse in the Anterior Chamber

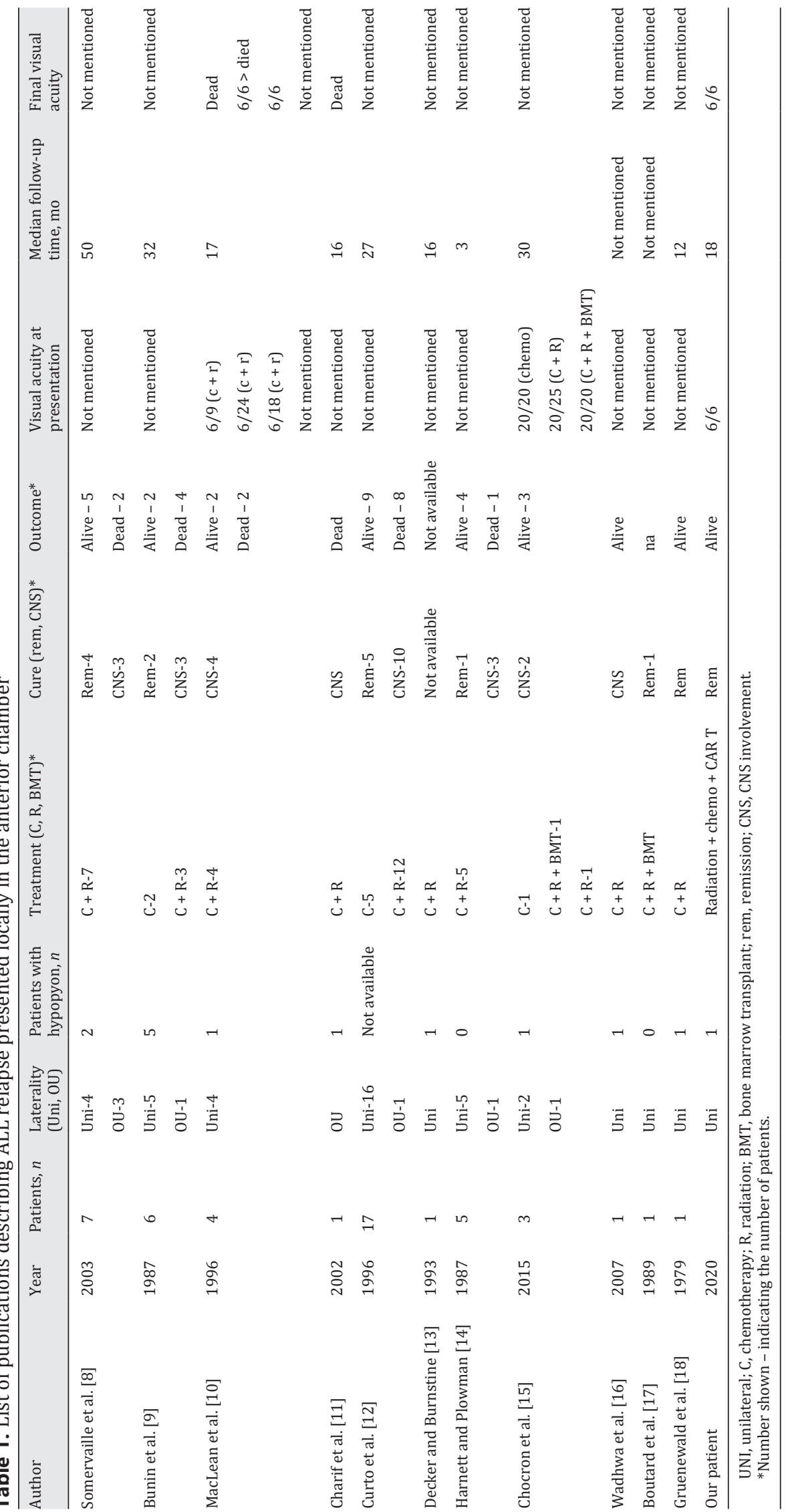


reported in most publications and were mentioned only in one report including 2 patients in whom visual acuity improved to 20/20 after receiving a combined treatment of chemotherapy and radiotherapy, but unfortunately one of them died after CNS recurrence. Our patient presented with a unilateral disease including malignant cells in the anterior chamber and a pseudo-hypopyon, 4 months after allogeneic hematopoietic cell transplantation. Treatment included 13 fractions of radiotherapy and the novel CAR T-cell therapy with cytarabine as a bridging chemotherapy. Follow-up time was 18 months.

Traditional treatments for ALL are intravenous chemotherapy, intrathecal chemotherapy, irradiation, and stem cell transplantation. CAR T-cell therapy is gaining popularity over the last decade, but is not used worldwide as standard of care [19]. To the best of our knowledge, no study, so far, has described the novel CAR T-cell treatment alone or in combination with radiotherapy for ocular ALL relapse. The result of the combined treatment of radiotherapy to the left eye and CAR T-cell therapy was excellent, with complete remission of the disease and a final visual acuity of 20/20. It is crucial to diagnose ALL relapse, and especially when systemic disease is yet to come. In a patient with a history of ALL who presents with abnormal ocular examination, with the presence of cells or "hypopyon" in the anterior chamber, a relapse of ALL must be considered and ruled out. Anterior chamber aspiration is useful in identifying malignant cells in the anterior chamber and in establishing the diagnosis [20-22]. Rapid diagnosis is crucial to enable an appropriate and fast treatment in order to control the disease and prevent further systemic involvement. The patient presented a complete ocular remission with no systemic or CNS involvement 18 months after the first presentation.

In conclusion, this is the first case of ALL relapse limited to the anterior chamber of the eye and treated with the novel CAR T-cell technology in combination with radiotherapy. On the last examination, 12 months after CAR T-cell therapy, the patient demonstrated outstanding ocular results; visual acuity was excellent in both eyes, and there was no ocular or systemic disease recurrence.

\section{Acknowledgment}

The authors would like to thank the photographer Galit Yair-Pur for the ocular pictures.

\section{Statement of Ethics}

This study protocol was reviewed and the need for approval was waived by the Helsinki Committee of the Tel Aviv Medical Center. Written informed consent was obtained from the patient for publication of this case report and any accompanying images.

\section{Conflict of Interest Statement}

The authors have no conflicts of interest to declare.

\section{Funding Sources}

The authors have no funding sources to declare.

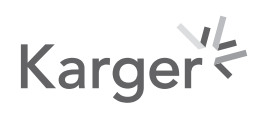




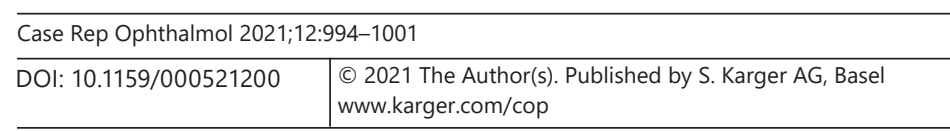

Gomel et al.: Chimeric Antigen Receptor T-Cell Therapy for ALL Relapse in the Anterior Chamber

\section{Author Contributions}

All authors took part in the treatment of the young boy and had contribution in writing and aprooving this case report.

\section{Data Availability Statement}

The data that support the findings of this study are not publicly available because they contain private information, but are available from the author upon reasonable request.

\section{References}

1 Nguyen K, Devidas M, Cheng SC, La M, Raetz EA, Carroll WL, et al. Factors influencing survival after relapse from acute lymphoblastic leukemia: a Children's Oncology Group study. Leukemia. 2008;22(12):2142-50.

2 Pui CH, Evans WE. Treatment of acute lymphoblastic leukemia. N Engl J Med. 2006;354(2):166-78.

3 Sharma T, Grewal J, Gupta S, Murray PI. Ophthalmic manifestations of acute leukaemias: the ophthalmologist's role. Eye. 2004;18(7):663-72.

4 Mateo J, Ascaso FJ, Nunez E, Peiro C, Gonzalez G, Cristóbal JA, et al. Ophthalmological manifestations in acute lymphoblastic leukemia. Novel aspects in acute lymphoblastic leukemia. InTech; 2011.

5 Ellis W, Little HL. Leukemic infiltration of the optic nerve head. Am J Ophthalmol. 1973;75(5):867-71.

6 Masera G, Carnelli V, Uderzo C, Toselli C, Lasagni F, Lambertenghi E. Leukaemic hypopyon in acute lymphoblastic leukaemia after interruption of treatment. Arch Dis Child. 1979;54(1):73-4.

7 Maude SL, Laetsch TW, Buechner J, Rives S, Boyer M, Bittencourt H, et al. Tisagenlecleucel in children and young adults with B-cell lymphoblastic leukemia. N Engl J Med. 2018;3785:439-48.

8 Somervaille TC, Hann IM, Harrison G, Eden TO, Gibson BE, Hill FG, et al. Intraocular relapse of childhood acute lymphoblastic leukaemia. Br J Haematol. 2003;121(2):280-6.

9 Bunin N, Rivera G, Goode F, Hustu HO. Ocular relapse in the anterior chamber in childhood acute lymphoblastic leukemia. J Clin Oncol. 1987;5(2):299-303.

10 MacLean H, Clarke MP, Strong NP, Kernahan J, Ashraf S. Primary ocular relapse in acute lymphoblastic leukemia. Eye. 1996;10(Pt 6):719-22.

11 Charif CM, Loughzail K, Benkirane N, Berraho A. Ocular relapse of acute lymphoblastic leukemia. Bull Soc Belge Ophtalmol. 2002;286:27-30.

12 Curto ML, D’Angelo P, Jankovic M, Fugardi MG, Ziino O, Casale F. Isolated ocular relapse in childhood acute lymphoblastic leukemia during continuing complete remission. Haematologica. 1996;81(1):47-50.

13 Decker EB, Burnstine RA. Leukemic relapse presenting as acute unilateral hypopyon in acute lymphocytic leukemia. Ann Ophthalmol. 1993;25(9):346-9.

14 Harnett AN, Plowman PN. The eye in acute leukaemia. 2. The management of solitary anterior chamber relapse. Radiother Oncol. 1987;10(3):203-7.

15 Chocron IM, Morrison DG, Friedman DL, Desai NA, Donahue SP. Ophthalmic manifestations of relapsing acute childhood leukemia. J AAPOS. 2015;19(3):284-6.

16 Wadhwa N, Vohra R, Shrey D, Iyer VK, Garg S. Unilateral hypopyon in a child as a first and sole presentation in relapsing acute lymphoblastic leukemia. Indian J Ophthalmol. 2007;55(3):223-4.

17 Boutard P, Reman O, Lemerle S, Guihard J. [A relapse in the eye of acute lymphoblastic leukemia]. Ann Pediatr. 1989;36(4):267-8.

18 Gruenewald RL, Perry MC, Henry PH. Leukemic iritis with hypopyon. Cancer. 1979;44(4):1511-3.

19 Xin Yu J, Hubbard-Lucey VM, Tang J. The global pipeline of cell therapies for cancer. Nat Rev Drug Discov. 2019; 18(11):821-2.

20 Abramson DH, Wachtel A, Watson CW, Jereb B, Wollner N. Leukemic hypopyon. J Pediatr Ophthalmol Strabismus. 1981;18(3):42-4.

21 Ninane J, Taylor D, Day S. The eye as a sanctuary in acute lymphoblastic leukaemia. Lancet. 1980;1(8166): 452-3.

22 Tate Holbrook C, Elsas FJ, Crist WM, Castleberry RP. Acute leukemia and hypopyon. J Pediatr. 1978;93(4): 626-8. 